\title{
Coulisses
}

Revue de théâtre

19 | Hiver 1999

Varia

\section{Bilan moral 1997-98, perspectives 1998-99}

Théâtre de la visibilité et/ou théâtre d'éveil

\section{Lucile Garbagnati}

\section{OpenEdition}

\section{Journals}

Édition électronique

URL : https://journals.openedition.org/coulisses/5588

DOI : $10.4000 /$ coulisses.5588

ISSN : 2546-9460

Éditeur

Presses universitaires de Franche-Comté

\section{Édition imprimée}

Date de publication : 1 janvier 1999

Pagination : $42-44$

ISBN : 2-913322-09-3

ISSN : 1150-594X

\section{Référence électronique}

Lucile Garbagnati, «Bilan moral 1997-98, perspectives 1998-99 », Coulisses [En ligne], 19 | Hiver 1999, mis en ligne le 18 octobre 2019, consulté le 12 janvier 2022. URL : http://journals.openedition.org/ coulisses/5588; DOI : https://doi.org/10.4000/coulisses.5588

Ce document a été généré automatiquement le 12 janvier 2022.

Coulisses 


\title{
Bilan moral 1997-98, perspectives 1998-99
}

\author{
Théâtre de la visibilité et/ou théâtre d'éveil ${ }^{1}$
}

Lucile Garbagnati

Tout d'abord, nous tenons à remercier tous ceux qui cette année encore nous ont fait confiance:

L'Université, en maintenant sa subvention, elle nous a permis de vivre, tant au sens propre qu'au sens figuré, puisqu'elle a ainsi renouvelé sa confiance dans le TU; le Conseil Régional, le Conseil Général, ce dernier a souligné la qualité de Coulisses ; la Mairie de Besançon, par ses aides diverses a manifesté l'intérêt qu'elle prenait aux activités $d u T U$ : production, action culturelle, recherche; le DUPM et l'Aire Urbaine 2000, qui s'efforce de créer sur un nouveau campus une vie culturelle digne des formations universitaires en place; en attribuant une bourse Culture Action a une étudiante impliquée dans la création collective Farces, le CROUS marque ainsi son intérêt pour une forme de production théâtrale qui met en scène un grand nombre d'étudiants et implique toute une formation à la fois intellectuelle et artistique; $l^{\prime} O T U$, pour la deuxième fois nous a accordé son mécénat. Il convient tout particulièrement de souligner la contribution des différents centres de recherche, le Centre Jacques-Petit, le Laboratoire d'histoire et des littératures des pays de langues européennes, l'École doctorale, qui reconnaissant le travail de recherche et de diffusion du TU en fait un membre de la communauté des chercheurs.

Nos remerciements s'adressent aussi à M. le Doyen de la Faculté des Lettres qui nous a accordé l'autorisation de répéter en soirée dans ses locaux.

\section{Préambule}

La suppression de plus de la moitié de la subvention de l'université en 1997 avait mis en péril l'existence même du TU. Une rallonge du CA lui avait permis de poursuivre ses activités. Il lui avait été reproché de ne pas être assez «visible», c'est à dire " médiatique "; Sans entrer dans une polémique vaine, on est en droit de se demander, une fois de plus, ce que l'on attend d'un "théâtre universitaire amateur »? d'être l'amuseur facile de la foule? d'être la troupe de l'université qui rassemble quelques étudiants sélectionnés pour un répertoire choisi? d'être le lieu de découverte, 
d'apprentissage pour tous et par tous d'un art qui unit le geste, le dire et le non-dit? C'est cette dernière voie que le TU a choisie, fidèle aux missions de l'université : Formation, Diffusion, Recherche. Si le TUFC devait trouver un nom ce serait non celui de « théâtre du visible » mais de « théâtre d'éveil ».

\section{Bilan : TU et « visibilité » \\ Des « Phynances " positives grâce aux remboursements des objecteurs et au CNASEA}

2 Les recettes globales s'élèvent à 506.740,17 francs. Mais il faut souligner qu'il y a 133.996,02 francs de produits exceptionnels : remboursements de soldes d'objecteurs, et du CNASEA au titre de l'emploi CES, ce qui réduit les recettes ordinaires à $372.744,15$ francs. Le contrat CES expire en décembre et nul ne sait s'il sera renouvelé dans les mêmes conditions. Ces fonds exceptionnels vont permettre d'engager comme salarié le secrétaire CES environ 72.000 francs (charges incluses), de réfléchir à un programme d'animation sur le pôle de Montbéliard dans le cadre de la Maison de l'Étudiant.

\section{Activités}

3 Le programme d'activités prévisionnel a été respecté et s'est efforcé de rendre le TU plus visible dans ses activités à la fois coutumières et spécifiques :

\section{Activités coutumières et visibilité}

\section{La diffusion de Naufrages : invitations et incitation au voyage}

4 Naufrages a été donné 9 fois, dont 3 fois au Petit Théâtre de La Bouloie, et a attiré un peu plus de 400 spectateurs, dont de nombreux invités, dont de nombreux membres des Conseils universitaires.

5 Pour la première fois, dans le cadre de la diffusion de Naufrages, nous avons emmené une étudiante non actrice de la pièce, adhérente du TU à Montbéliard. Hispanisante en LEA elle a pu expérimenter ses talents de traductrice à Lugo, ville du festival déconcentré de Saint Jacques de Compostelle. Cette initiative a permis à une étudiante de "sortir de chez elle». Elle s'inscrit comme une des modalités d'action évoquée ultérieurement dans l'étude de Guy Barbier sur la vie culturelle des étudiants du pôle universitaire Belfort-Montbéliard. (Voir ci-après.) Elle est à renouveler en fonction de la langue nationale du festival.

\section{Farces : repas farcesques aux $R U$, questionnaire}

6 La production de Farces dans le cadre des Rencontres Jeune Création a été un succès (environ 300 spectateurs pour une seule représentation, dont de nombreux invités membres des Conseils de l'Université). Elle a été annoncée par un menu farcesque avec la participation des RU. Malheureusement, la publicité a été insuffisante. Mais l'idée est à reprendre et à approfondir. Un questionnaire distribué à l'issue du spectacle donne un aperçu de la composition du public du TU et de ses réactions. ${ }^{2}$ 


\section{Nouveautés}

\section{L'autocar-découverte}

7 II s'agit moins d'une nouveauté que d'une reprise. Le succès de l'autocar pour le spectacle d'Ariane Mnouchkine Et soudain des nuits d'éveil, nous a donné envie de reprendre cette activité que nous avions abandonnée. Au-delà de la découverte d'un lieu et d'un spectacle, la manifestation permet de faire connaître le TU à d'autres personnes que les adhérents. Nous proposons donc de découvrir, l'avant-dernière représentation mondiale de la mise en scène mythique de Strehler d'Arlequin, serviteur de deux maîtres, à Dijon le 29 janvier 1999.

\section{Coulisses et les PUFC}

8 A l'occasion de la structuration des publications de l'Université, Coulisses a été admis aux Presses Universitaires Franc-Comtoises dans la série « Revues ». La revue bénéficie des avantages des PUFC: adhésion au collectif des éditeurs franc-comtois Les Nompareilles, participation aux Salons du livre de Paris, de Lausanne, inscription sur le catalogue. Coulisses y gagne ainsi en notoriété et en visibilité.

\section{Visibilité et participation à la vie universitaire}

9 Le TU a participé aux Journées Portes Ouvertes de l'Université par des interventions théâtrales dans les bus; il a animé une émission bimensuelle d'une heure sur Radio Campus.

\section{Le Colloque " Théâtre et Sciences " : théorie et spectacles}

10 Il a réuni une trentaine de chercheurs et des auditeurs en nombre variable. Les Actes témoignent de la richesse des approches transdisciplinaires, et de l'immensité d'un champ de recherche quasiment vierge. Les spectacles ont permis à un colloque universitaire de sortir des murs de l'université et d'établir une forme de co-production avec le Service culturel du CROUS et le Théâtre de l'Espace, scène nationale, qui sont prêts à recommencer l'expérience.

\section{Culture, visibilité, moyens}

11 Si en terme d'activités le bilan peut être considéré comme très satisfaisant, il est peutêtre moins positif en termes de visibilité. En effet, les initiatives, repas farcesques, improvisations dans les bus, émissions sur Radio Campus n'ont pu avoir lieu que grâce à une stagiaire, en contrat déterminé de six mois. Qui va reprendre le flambeau? Avec quel budget? Une fois de plus, on se retrouve face à la question des moyens. En effet, une action sur la durée ne peut être envisagée qu'avec un personnel stable qui acquiert de l'expérience et peut faire progresser l'association au lieu qu'elle soit le siège de perpétuels recommencements. 


\section{Perspectives}

\section{Programme} Théâtre Université abandonnées pour raisons financières en 1997. Elles seront l'occasion d'un nouveau partenariat avec le Service culturel du CROUS, et d'une manifestation commune avec le TU à Montbéliard en fonction des langues des troupes retenues.

\section{Les 10 ans de Coulisses}

En mai Coulisses publiera son $20^{\mathrm{e}}$ numéro et fêtera ses 10 ans ! Sous quelle forme?

18 Nouvelle présentation? Numéro spécial? Publication d'un texte de théâtre inédit? Affaire en cours! Qu'on se le dise! Que tous ceux qui sont intéressés n'hésitent pas à nous contacter! 


\section{L'avenir du TUFC}

19 Au-delà de ses activités, il s'agit de réfléchir à la place que doit tenir le TU dans le cadre du plan quadriennal de l'Université, et plus généralement dans U.3000. Quelques pistes sont d'ores et déjà à suivre.

\section{Le plan quadriennal et la Maison de l'Étudiant à Besançon}

20 Il s'agit de faire figurer les besoins du TU et d'obtenir leur inscription dans le plan quadriennal. Comment faire admettre que le TU a besoin d'un lieu de répétition permanent d'environ $200 \mathrm{~m}^{2}$, vide, et que sans ce lieu à la fois technique et identitaire, il risque de s'étioler. Quel statut stable qui lui permette de poursuivre et d'approfondir ses activités?

\section{Quel programme pour la Maison de l'Étudiant à Montbéliard ?}

21 Il s'agit de réfléchir à un programme d'animation sur le pôle de Montbéliard dans le cadre de la Maison de l'Étudiant.

22 Mais «II n'est pas nécessaire d'espérer pour entreprendre, ni de réussir pour persévérer ». C'est peut-être là le ressort nécessaire pour que se réalise l'utopie du théâtre.

\section{NOTES}

1. Ce texte, actualisé, est celui qui a été remis au Conseil d'Administration du TUFC le 7 octobre 1998.

2. Voir p. 40 pour les résultats de ce questionnaire. 